\title{
DHA Hydroperoxides as a Potential Inducer of Neuronal Cell Death: a Mitochondrial Dysfunction-Mediated Pathway
}

\author{
Xuebo Liu ${ }^{1}$, Takahiro Shibata ${ }^{1}$, Shinsuke Hisaka ${ }^{1}$, Yoshichika Kawai ${ }^{1,2}$, and Toshihiko Osawa ${ }^{1, *}$ \\ ${ }^{1}$ Laboratory of Food and Biodynamics, Graduate School of Bioagricultural Science, Nagoya University, Nagoya \\ 464-8601, Japan \\ ${ }^{2}$ Department of Food Science, Graduate School of Nutrition and Bioscience, The University of Tokushima, \\ Tokushima 770-8501, Japan
}

Received 25 December, 2007; Accepted 29 January, 2008

\begin{abstract}
Summary During the lipid peroxidation reaction, lipid hydroperoxides are formed as primary products. Several lines of evidence suggest that lipid hydroperoxides can trigger cell death in many cell types, including neurons. In a screening of lipid hydroperoxides which can induce toxicity in neuronal cells, we found docosahexaenoic acid hydroperoxides (DHA-OOH) induced much severe levels of reactive oxygen species generation and cell death in human neuroblastoma SH-SY5Y cells compared to the hydroperoxides of linoleic acid and arachidonic acid. Therefore, we focused on DHA-OOH, and demonstrated that DHA-OOH apparently induced an apoptosis in the neuronal cells through several apoptotic hallmarks including nuclei condensation, DNA fragmentation, poly (ADP-ribose) polymerase cleavage and increased activity of caspase-3. We also found the signaling changes in mitochondriamediated apoptosis, such as cytochrome $\mathrm{c}$ release and increased expression of Bcl-2, as well as a dose-dependent attenuation of mitochondrial membrane potential in the DHA-OOH treated cells. These data indicated DHA hydroperoxide as a potential inducer of apoptosis in human neuroblastoma SH-SY5Y cells, which may be mediated by mitochondria dysfunction pathway.
\end{abstract}

Key Words: lipid peroxidation, DHA hydroperoxides, apoptosis, mitochondria dysfunction, neurodegeneration

\section{Introduction}

Polyunsaturated fatty acids (PUFA), such as linoleic acid $(\mathrm{C} 18: 2, \omega 6)(\mathrm{LA})$, arachidonic acid $(\mathrm{C} 20: 4, \omega 6)(\mathrm{AA})$ and docosahexaenoic acid (C22:6, $\omega 3)$ (DHA), are essential for the maintenance of the normal biological functions; however, they are highly unsaturated and, therefore, are the most vulnerable for free radical damage termed lipid peroxidation [1]. Lipid peroxidation in tissue and in tissue fractions represents a degradative process, has been implicated in the pathogenesis of numerous diseases, such as atherosclerosis,

\footnotetext{
*To whom correspondence should be addressed.

Tel: +81-52-789-4125 Fax: +81-52-789-5741

E-mail: osawat@agr.nagoya-u.ac.jp
}

diabetes, cancer, as well as several neurodegenerative diseases [2]. During the lipid peroxidation, lipid hydroperoxides are formed as primary products. It has been reported that lipid hydroperoxides also possess highly reactive biological properties like as subsequent secondary product aldehydes, both of which can react on proteins to form adducts, alter cellular responses, and induce apoptosis in many cell lines [3-6].

In the brain, a high concentration of polyunsaturated fatty acids, especially DHA and AA, are implicated relative to other organs, therefore, lipid peroxidation is one of the major outcomes of free radical-mediated injury to brain, where it directly damages membranes and generates a number of oxidized products. In our previous report, we have in vivo described the formation of a DHA hydroperoxides-derived protein modified adduct $N^{\varepsilon}$-(succinyl) lysine (SUL); more- 
over, the formation of SUL was then immunochemically demonstrated in the liver of mice fed with DHA followed by intraperitoneal injection of carbon tetrachloride (CCl4), a hepatic lipid peroxidation model [4]. In addition, Nathalie Bernoud-Hubac et al. have identified. $\gamma$-ketoaldehyde isoketals (Isoks) derived from AA and neuroketals (NeuroKs) derived from DHA [7]. Although the pathophysiological consequence of the overproduction of the lipid peroxidative products have been highlighted in brain tissue, it is noteworthy that these reactive products are also generated at low levels in neuronal membrane and appear to play a role in physiological signaling of neuron, such as apoptosis [8].

Apoptosis has been considered the main cause of neuronal death in some human neurodegenerative disorders, such as Alzheimer disease (AD) [9]. In recent years, many reports have focused on aldehydes to investigate the effect of lipid peroxidation on apoptosis in neuronal cells, whereas although little was known, the primary products of lipid peroxidation, hydroperoxides, may also be greatly account for the disorders of neuronal cells. In the present study, through a screening we found that DHA hydroperoxides (DHA-OOH) showed a much severe toxic effect to neuronal cells, thus, we focused on DHA-OOH to investigate whether and how it induced an apoptotic cell death in human neuroblstoma SH-SY5Y cells. The exploration may provide new clues to the pathogenic mechanism in neurodegenerative diseases.

\section{Materials and Methods}

\section{Materials}

DHA (purity $\geq 98 \%$ ) was purchased from the Cayman Chemical Co. (Ann Arbor, MI). Arachidonic acid and $\alpha-$ linolenic acid were obtained from the Sigma-Aldrich Co. (St. Louis, MO). The antibodies against poly (ADP-ribose) polymerase (PARP) were purchased from the Cell Signaling Technology, Inc. (Beverly, MA). The antibodies against Bcl-2 (100) and Bax were obtained from the Santa Cruz Biotechnology (Santa Cruz, CA). Purified Mouse AntiCaspase-3 (CPP32, not recognizing cleaved form of caspase-3 in the western blot) and purified Mouse Anticytochrome $\mathrm{c}$ were obtained from BD Pharmingen (Franklin Lakes, NJ). 13-Hydroperoxy-octadecadienoic acid (13HPODE) and 15-hydroxyeicosatetraenoic acid (15-HPETE) were enzymatically synthesized from linoleate and arachidonic acid, respectively, by soybean lipoxygenase as previously described $[10,11]$.

\section{DHA hydroperoxides preparation}

The DHA-OOH was prepared by the reaction of lipoxidase (from soybean, Wako Pure Chemical Industries, Ltd., City, Japan) with docosahexaenioc acid as previously de- scribed [12]. The reaction mixture containing $83.6 \mathrm{mg}$ docosahexaenoic acid, $16 \mathrm{mg}$ lipoxidase and $220 \mathrm{ml}$ of borate buffer ( $200 \mathrm{mM}, \mathrm{pH} 9.0)$ were used, and the reaction was carried out in a flask filled with oxygen at room temperature. After incubation for $10 \mathrm{~min}$, to terminate the reaction, $\mathrm{HCL}$ was added to the mixture till the solution $\mathrm{pH}$ was below 4.0. The formed hydroperoides were extracted twice with an equal amount of chloroform/methanol (1:1), and the collected chloroform layer was then evaporated. The obtained DHA-OOH was dissolved in ethanol. The identification was performed by HPLC analysis monitored at $\mathrm{A}_{234}$ and the concentration was quantified using a lipid hydroperoxide kit (Cayman, Ann Arbor, Michigan) compared to a standard curve prepared by authentic 13-HPODE.

\section{3-HPODE preparation}

The hydroperoxide of linoleic acid, 13-HPOD, was made from linoleic acid by soybean lipoxygenase (SLO) as previously described [10]. The linoleic acid $(0.7 \mathrm{mg} / \mathrm{ml})$ was dissolved in $0.1 \mathrm{M}$ borate buffer ( $\mathrm{pH} 9.0$ ). SLO (7600 unit/ $\mathrm{ml}$ ) was added to the solution and reacted under $\mathrm{O} 2$ with stirring at room temperature. After $90 \mathrm{~min}$, the $\mathrm{pH}$ was adjusted to 4.0 with $0.1 \mathrm{M} \mathrm{HCl}$ for the termination of the oxidation. The peroxide formed was extracted with a 2-fold volume of chloroform-methanol 1:1. The extract was evaporated, dissolved in chloroform, and then applied to a thinlayer chromatography (TLC) plate. The chromatography was performed using a development with n-hexane-diethyl ether 4:6. The band of 13-HPODE was visualized by a $\mathrm{W}$ lamp and scraped off. The 13-HPODE was extracted with chloroform andethanol and stored under argon at $-70^{\circ} \mathrm{C}$ or $-20^{\circ} \mathrm{C}$ before use. The identification was performed on the basis of 'H-nuclear magnetic resonance spectrometry and coelution of HPLC with authentic 13-HPODE commercially obtained. The concentration of 13-HPODE was determined by absorbance at $234 \mathrm{~nm}$ using $\mathrm{E}=25000 \mathrm{M}-\mathrm{c}^{-} \mathrm{cm}-'$.

\section{5-HPETE preparation}

15-HPETE was synthesized from $25 \mu \mathrm{M}$ of arachidonic acid in $30 \mathrm{ml}$ of $0.1 \mathrm{M}$ Tris, $\mathrm{pH} 9.0$ that had been saturated with oxygen as described in reference 11 . The reaction was initiated by adding $0.25 \times 10^{6} \mathrm{U}$ of 15 -lipoxygenase and proceeded for $2 \mathrm{~min}$ at room temperature. Subsequently, a second aliquot of 15-lipoxygenase $\left(0.25 \times 10^{6} \mathrm{U}\right)$ was added, and the reaction continued for an additional $8 \mathrm{~min}$. Oxygen was gently bubbled into the buffer throughout the reaction. Formation of 15-HPETE was monitored by an increase in $A_{235}$. The reaction was stopped by adding $7.5 \mathrm{ml}$ of ethanol and chilling on ice. After acidification to $\mathrm{pH} 3$ with $12 \mathrm{~N} \mathrm{HCl}$, lipids were extracted three times with 4 volumes of ethyl acetate/hexane (1:1). The combined organic layers were dried over anhydrous sodium sulfate and concentrated by a stream of nitrogen gas. The extracts 
A

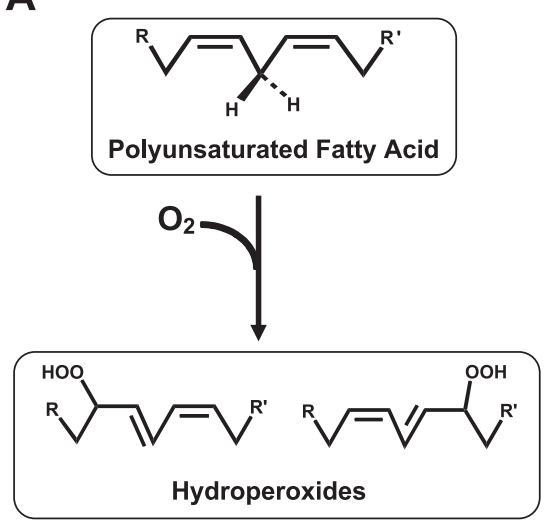

B
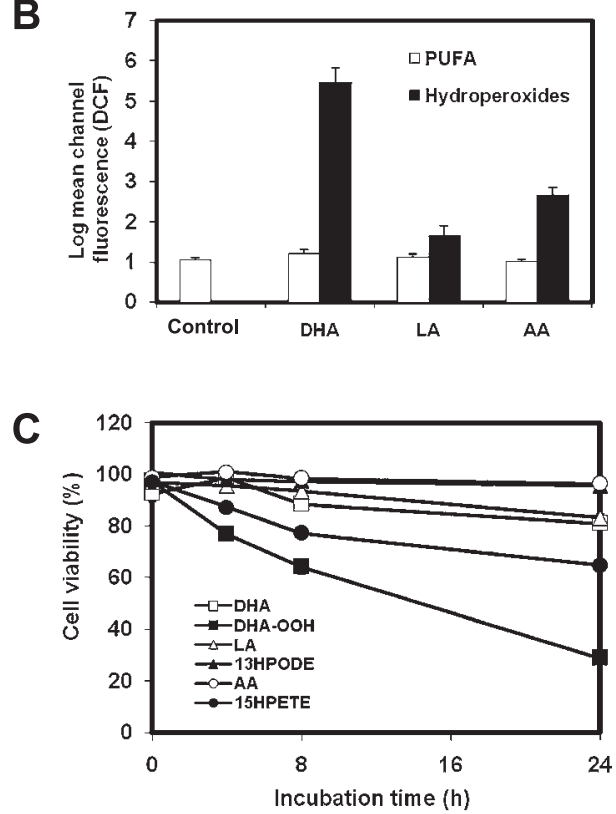

Fig. 1. ROS generation and cell viability. A, Scheme for the formation of lipid hydroperoxides. B, ROS generation. The cells were loaded with carboxy-H2DCFDA for $30 \mathrm{~min}$, followed by exposure to $10 \mu \mathrm{M}$ PUFA or lipid hydroperoxides for $45 \mathrm{~min}$. The carboxy- $\mathrm{H}_{2}$ DCFDA-loaded cells without any treatment were used as the control. C, Cytotoxicity. The cells were exposed to $10 \mu \mathrm{M}$ of different agents for 0-24 h. Cell viability was measured by the MTT assay. In the MTT assay, data are expressed as \% of control culture conditions. Open square, DHA; closed square, DHA-OOH; open triangle, linoleic acid (LA); closed triangle, 13-HPODE; open circle, Arachidonic acid (AA); closed circle, 15-HPETE.

were applied to a silica HPLC semi-preparative column (Waters Spherisorb S10 W, $5 \mu \mathrm{m}, 10 \times 250 \mathrm{~mm}$ ) and eluted isocratically with hexane/isopropanol/acetic acid (985:15:1, $v / v / v$ ) at a flow rate of $2.0 \mathrm{ml} / \mathrm{min}$; the eluate was monitored by $A_{234}$. Fractions containing 15 -HPETE were combined, concentrated by evaporation with a stream of nitrogen gas and stored at $-20^{\circ} \mathrm{C}$. The concentration of 15 -HPETE was determined using $\varepsilon 234=23 \mathrm{mM}^{-1} \mathrm{~cm}^{-1}$.

\section{Cell culture and cell viability}

Human neuroblastoma SH-SY5Y cells were grown in Cosmedium-001 (Cosmo-Bio, Tokyo, Japan) containing 5\% fetal bovine serum. The cells were seeded in plates coated with polylysine and cultured at $37^{\circ} \mathrm{C}$. The cell viability was quantified by MTT assay.

\section{Analysis of reactive oxygen species (ROS) production}

The endogenous ROS level was detected by flow cytometry using $\mathrm{H}_{2} \mathrm{DCF}-\mathrm{DA}$ as previously described [12]. In the present study, the SH-SY5Y cells were treated with DHA-OOH $(10 \mu \mathrm{M})$ for $30 \mathrm{~min}$, followed by staining with DCHF-DA (100 uM) for an additional $30 \mathrm{~min}$. The fluorescence of dichlorofluorescein (DCF) in the supernatant was measured by an EPICS Elite Flow Cytometer.

\section{Nuclear staining assay}

To determine the apoptotic nuclei, cells treated with $10 \mu \mathrm{M}$ DHA-OOH for $4 \mathrm{~h}$ were stained with a fluorescent DNA-binding dye, Hoechst 33258, and observed using a fluorescence microscope (Olympus Optical Co., Ltd., Tokyo, Japan).

\section{Analysis of DNA fragmentation}

For analysis of the DNA fragmentation by agarose gel electrophoresis, the cellular DNA was extracted from whole cells treated with $10 \mu \mathrm{M}$ DHA-OOH. Analysis of DNA fragmentation was performed as described previously [3].

\section{Subcellular fraction of SH-SY5Y cells}

Subcellular isolation from the cells was carried out as described by Pallotti et al. [13]. Briefly, cells are harvested by centrifugation at $600 \times g$ for $10 \mathrm{~min}$, washed with PBS, and resuspended with 5 volumes of Solution A $(0.25 \mathrm{M}$ sucrose, $20 \mathrm{mM}$ HEPES-KOH, pH 7.5, $10 \mathrm{mM} \mathrm{KCl,} 1.5 \mathrm{mM}$ $\mathrm{MgCl}_{2}$, $1 \mathrm{mM}$ EDTA, $1 \mathrm{mM}$ EGTA, $1 \mathrm{mM}$ dithiothreitol, $0.1 \mathrm{mM}$ PMSF). The cellular suspension was homogenized with a glass-glass homogenizer with 20 up and down passes of the pestle. The homogenate was then centrifuged at $750 \times g$ for $10 \mathrm{~min}$. The resulting supernatant was collected, and then centrifuged at $10,000 \times g$ for $15 \mathrm{~min}$. The supernatant was used as the cytosol fraction. 
A
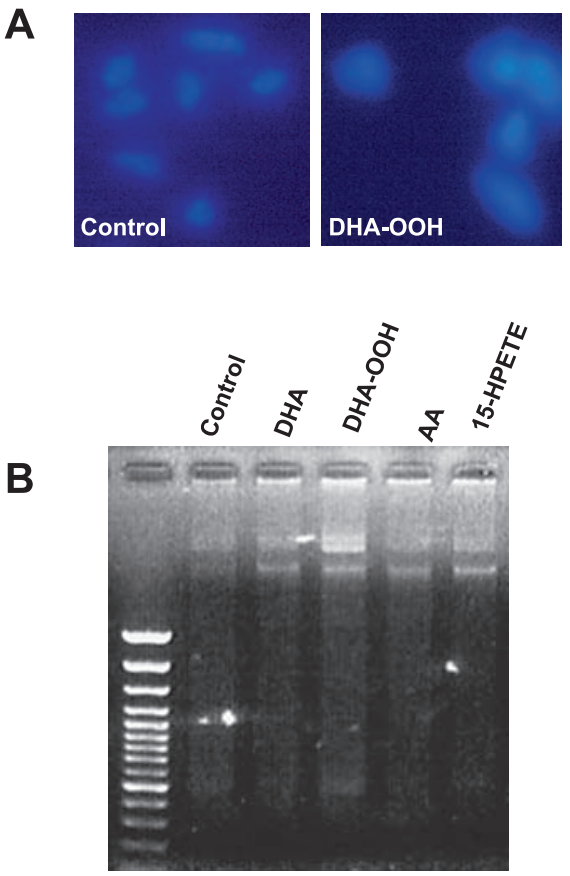

C

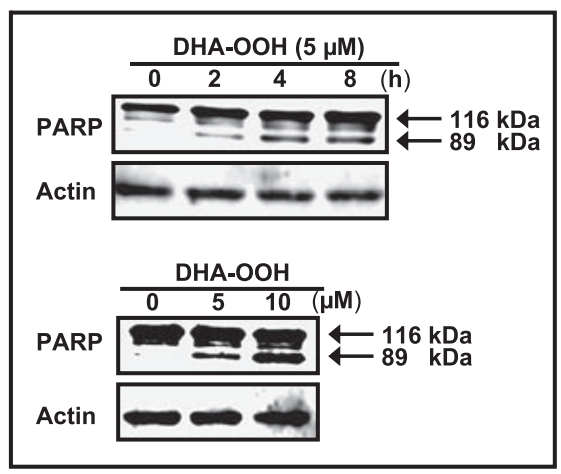

D

Fig. 2. Induction of apoptotic cell death. A, Nuclei condensation in SH-SY5Y cells exposed to $10 \mu \mathrm{M}$ DHA-OOH. SH-SY5Y cells were stained with Hoechst 33258, and examined by fluorescence microscopy. Left panel, control. Right panel, DHA-OOH treatment. B, DNA fragmentation in SH-SY5Y cells exposed to $10 \mu \mathrm{M}$ DHA-OOH for $4 \mathrm{~h}$. Nucleosomal DNA fragmentation was visualized by agarose gel electrophoresis. C, PARP cleavage in SH-SY5Y cells exposed to DHA-OOH. The cleavage of PARP was analyzed by Western blotting. Upper panel, time-dependent cleavage of PARP. Lower panel, dose-dependent cleavage. $\mathrm{D}$, Analysis of caspase-3 expression. The cells were treated with $10 \mu \mathrm{M}$ DHA-OOH for $0-8 \mathrm{~h}$. Expression of pro-caspase 3 was detected by western blotting using caspase-3 antibody (not recognizing cleaved form of caspase- 3 in the western blot).

\section{Western blot analysis}

The homogenates prepared from the cells were treated with the SDS sample buffer and then immediately boiled for $5 \mathrm{~min}$. The protein concentrations were determined using the BCA protein assay kit (Pierce, Rockford, USA). The proteins were separated by SDS-PAGE in the presence of 2-mercaptoethanol and electrotransferred onto a nitrocellulose membrane (Hybond ECL) (Amersham Biosciences, Tokyo, Japan). To detect the immunoreactive proteins, we used horseradish peroxidase-conjugated anti-rabbit or mouse or goat IgG and ECL blotting reagents (Amersham Biosciences, Tokyo, Japan).

\section{Measurement of the mitochondrial membrane potential}

The mitochondrial membrane potential was measured as described by Ko et al. [14]. Briefly, SH-SY5Y cells after different treatments were incubated with $50 \mathrm{nM}$ DiOC6 for $30 \mathrm{~min}$ at $37^{\circ} \mathrm{C}$, followed by washing with ice-cold PBS, after which the cell pellets were collected. The pellets were suspended in $600 \mu \mathrm{l}$ of PBS, and the fluorescent intensities of the cells were analyzed using an EPICS Elite Flow Cytometer (Beckman Coulter, Inc., Fullerton, CA) at the respective wavelengths for excitation and emission of 484 and $500 \mathrm{~nm}$.

\section{Results}

\section{ROS generation and cell viability}

To identify what hydroperoxides can induce much severe toxicity in neuronal cells, we conducted a screening of the lipid hydroperoxides derived from DHA, AA and LA, respectively, for ROS generation and cell viability in neuroblastoma SH-SY5Y cells. As shown in Fig. 1, after treatment with $10 \mu \mathrm{M}$ of different agents for $30 \mathrm{~min}$, significant ROS generation induced by DHA-OOH was found compared to that treated by 13-HPODE and 15-HPETE which are major hydroperoxides derived from linoleic acid and arachidonic acid, respectively (Fig. 1B). Moreover, we measured the numbers of viable cells under exposure to the hydroperoxides by MTT assay. Likewise, DHA-OOH induced an apparent time-dependent decrease in the viable cells at a concentration at $10 \mu \mathrm{M}$, whereas no significant cell death was found up to $24 \mathrm{~h}$ when the cells were treated with the same dose of 15-HPETE and 13-HPODE (Fig. 1C). In addition, in both the ROS generation and cell death cases, polyunsaturated fatty acids themselves almost exhibited no effect on the neuronal cells. 
A

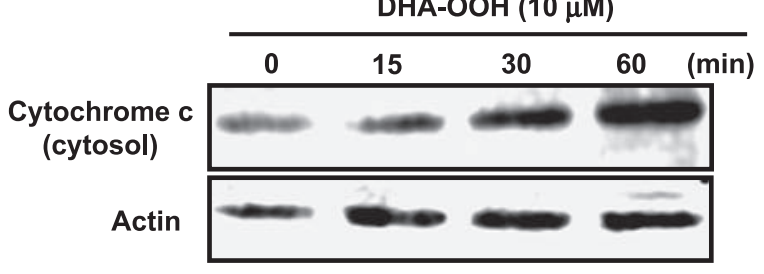

B

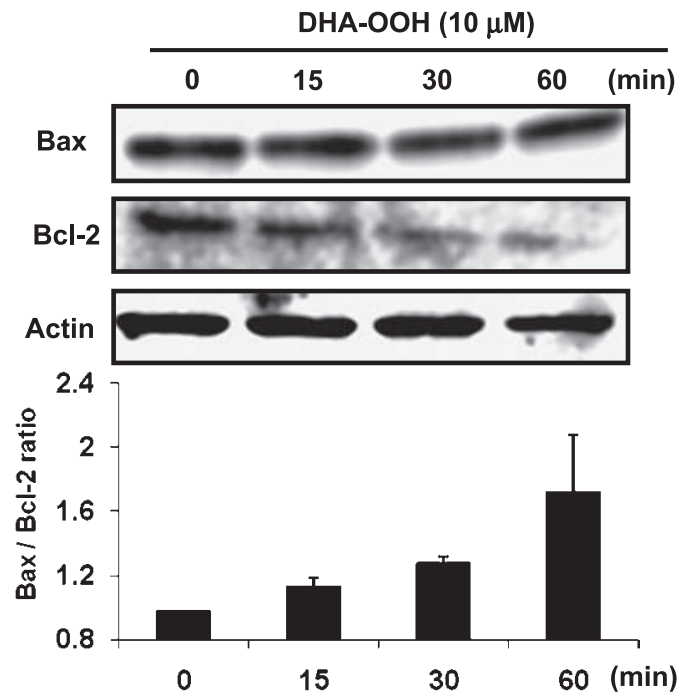

Fig. 3. The increased cytochrome c release and Bax/Bcl-2 ratio in response to DHA-OOH. A, Cytochrome c release. SH-SY5Y cells were treated with DHA-OOH (10 uM) for different times $(0,15,30$ and $60 \mathrm{~min})$, and the cytosolic fraction were prepared. The expression of cytochrome $\mathrm{c}$ in the fraction was detected by western blotting. B, Expression of Bcl-2 family protein. The whole lysates extracted from the DHA-OOH-treated cells were used to analyze the expression of Bcl-2 family protein by western blotting, and the ratio of $\mathrm{Bax} / \mathrm{Bcl}-2$ was analyzed. The results are the means of three experiments.

\section{Induction of apoptotic cell death}

Based on the identification that DHA-OOH significantly led to a time-dependent decrease in the number of viable cells (Fig. 1C), next we focused on DHA-OOH to characterize whether DHA-OOH-induced cell death included apoptosis in human neuroblastoma SH-SY5Y cells. When human nueroblastoma SH-SY5Y cells were exposed to $10 \mu \mathrm{M}$ DHA-OOH for $4 \mathrm{~h}$, nuclei condensation was found in cells implicating a typical morphological feature of apoptosis (Fig. 2A). The gel electrophoresis of DNA from SH-SY5Y cells treated with DHA-OOH also displayed nucleosomal DNA fragmentation (Fig. 2B). Moreover, as hallmarks of apoptosis, a dose and time- dependent proteolytic cleavage of PARP resulting in the accumulation of the $89-\mathrm{kDa}$ (Fig. 2C), as well as a time-dependent decrease in the pro-caspase 3 expression (Fig. 2D), was seen in the cells after the treatment with DHA-OOH, thus indicating that DHA-OOH induced an apoptosis in the neuronal cells.

\section{Cytochrome c release and Bax/Bcl-2 ratio increased in response to $\mathrm{DHA}-\mathrm{OOH}$}

The release of cytochrome c (Cyt c) from mitochondria into the cytosol, followed by activity of caspase 3, has been identified in the process of mitochondria-dependent apoptosis. On the other hand, Bcl-2 family proteins have been shown to be involved in Cyt $\mathrm{c}$ release through the increasing translocation of the pro-apoptotic Bcl-2 family proteins Bax to the mitochondria [15]. In this study, to determine whether DHA-OOH-induced apoptosis is medicated by a mitochondrial pathway, we examined the expression levels of Cyt $\mathrm{c}$ in the cytosol fraction, as well as Bcl-2 and Bax in the whole cell lysates. As shown in Fig. 3A, treatment of the cells with $10 \mu \mathrm{M}$ DHA-OOH cause a significant increase in the Cyt c levels in the cytosol fraction for $60 \mathrm{~min}$. In addition, in this study, although significant changes in the expression of Bax in the DHA-OOH-treated cells were not observed, a time-dependent decrease in the expression levels of $\mathrm{Bcl}-2$ was found. Thus, an apparent increase in the $\mathrm{Bax} / \mathrm{Bcl}-2$ ratio was determined by a densitometric analysis of the bands (Fig. 3B), which is thought to be a key hallmark for the execution of apoptosis. These results suggest that the apoptotic cell death induced by DHA-OOH in SH-SY5Y cells may be mediated via the mitochondria mediated pathway.

\section{Loss of mitochondrial membrane potential}

The involvement of mitochondria in the DHA-OOH induced apoptosis process in SH-SY5Y cells focused our attention on what has happened in the mitochondria in the apoptotic cells, and thus we examined the function of mitochondria in the DHA-OOH-treated cells. The mitochondrial membrane potential was measured by flow cytometry using $\mathrm{DiOC}_{6}$ as a fluorescent dye. The data in Fig. 4A showed a dose-dependent decrease in the mitochondrial membrane potential in the DHA-OOH-treated SH-SY5Y cells, in contrast, almost no attenuation of mitochondria membrane potentials were detected in the 13-HPODE or 15-HPETEtreated cells (Fig. 4B).

\section{Discussion}

It has long been thought that oxidative stress is involved in the pathogenesis of neurodegenerative diseases including $\mathrm{AD}$ and Parkinson diseases (PD) [16-19]. Recently, much of the investigation has fueled interest in PUFA oxidation in the molecular pathogenesis of the diseases, especially AA $(20: 4, \omega 6)$ and DHA $(22: 6, \omega 3)$, which are evenly 

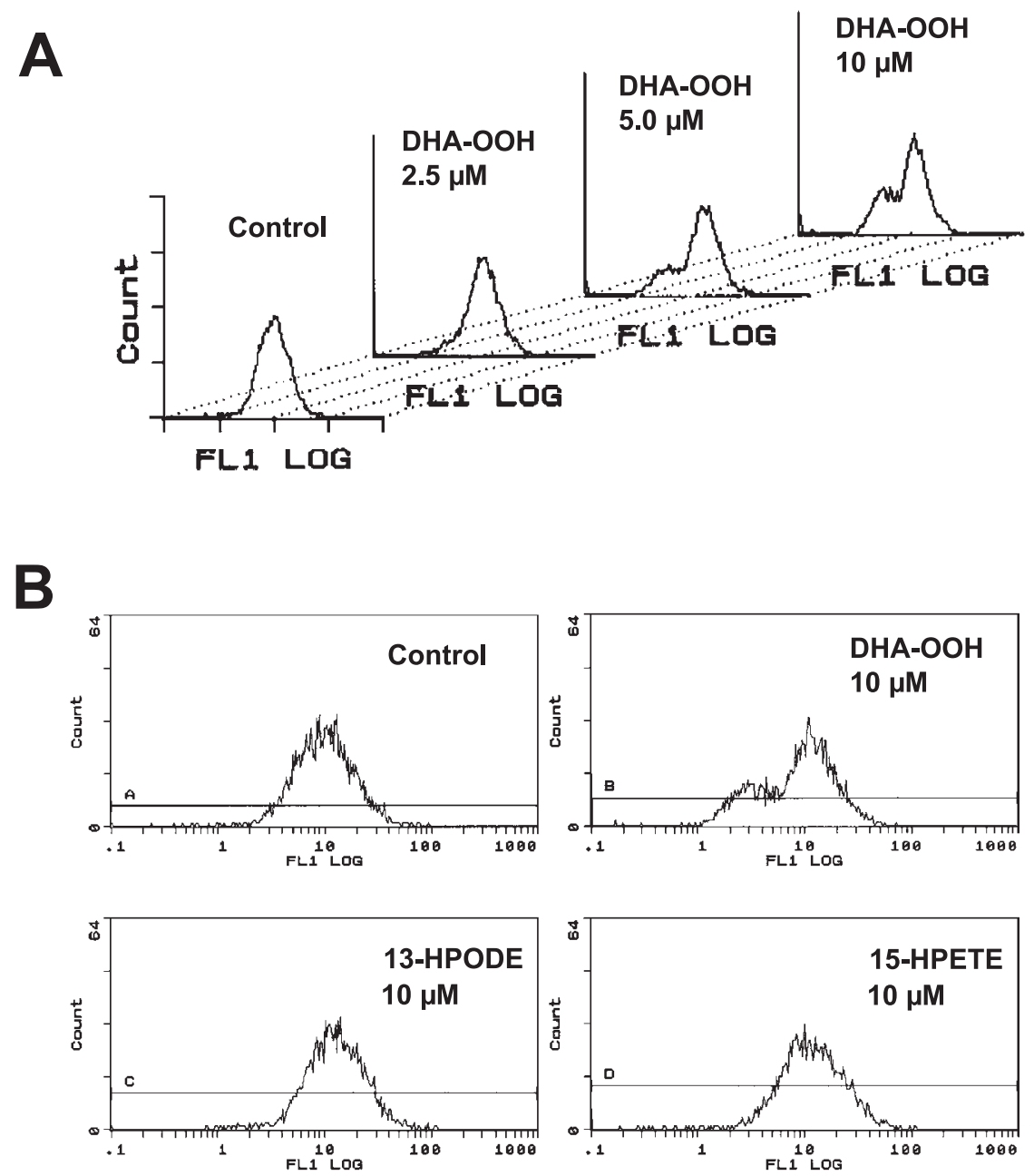

Fig. 4. Loss of mitochondrial membrane potential. A, SH-SY5Y cells were treated with different concentration of DHA-OOH (0, 2.5, $5,10 \mu \mathrm{M})$. DiOC 6 was added to the culture medium for a further $30 \mathrm{~min}$. The fluorescence intensity of DiOC 6 in cells was analyzed by flow cytometry. B, As described in A, the effect of 13-HPODE and 15-HPETE to mitochondria membrane potential were also measured compared to DHA-OOH.

distributed in the gray matter and white matter and enriched in neuronal membranes, respectively $[20,21]$. Regardless of the oxidative damage of aldehydes to neuronal cells, the primary product during PUFA oxidation, hydroperoxides, have also been reported having a high biologic activity that affects bodily functions [22]. For instance, AA-derived hydroperoxide 15-HPETE induces apoptosis in human neuroblastoma CHP 100 cells by increasing intracellular calcium [23]. In this study, through a screening we found that DHA-OOH showed a much severe neurotoxic effect, including ROS generation and cell death induction, compared to that of the hydroperoixdes derived from arachidonic acid and linoleic acid. Additionally, it is believed that the equal amounts of -OOH included in DHA$\mathrm{OOH}, 15$-HPETE and 13-HPODE were added to the cells because the concentration of the hydperoxides were commuted prior to the administrations by lipid hydro- peroxide kit (Cayman) compared to a standard curve prepared by authentic 13-HPODE.

In the following investigation that focused on DHA$\mathrm{OOH}$-induced neuronal cell death, we indicate that DHA$\mathrm{OOH}$ induced an apoptosis in SH-SY5Y cells, and suggest the mechanism might be mediated by a mitochondria dysfunction pathway. The results agree with Zhu et al. who suggest that mitochondrial dysfunction may play very important roles in neuronal apoptosis [24]. Moreover, it is well known that mitochondria mediated neuronal apoptosis is a common mechanism of cell loss in several neurodegenerative diseases, therefore, due to the high concentration of DHA in the brain and highly susceptible to oxidative stress, DHA-OOH-induced apoptosis may be much involved in the pathogenesis of neurodegenerative diseases.

DHA is well known as the main polyunsaturated fatty acid enriched in the central system, especially, the brain. In 
recent years, many papers have reported its beneficial physiological effects, such as learning ability, and hypolipidemic and neuroprotective effects [25-28]. However, DHA is highly unsaturated and, therefore, is the most vulnerable target for free radical damage termed lipid peroxidation [1]. It has been implicated that DHA concentrations decreased in the neurodegenerative brain [29], although the precise mechanism for the decrease is not well understood, it has been suggested that the lipid peroxidation of DHA is associated with the loss due to the increased detection of DHA peroxidative products in the patient's brain, such as 4-hydroxy-2-hexenal and NeuroKs [7, 30]. Despite the fact that DHA peroxidation is a significant causal factor being responsible for the decrease in the DHA concentration, on the other hand, it is possible that the products of the DHA peroxidation may play some damaging role in the neural systems, but as to this aspect little has been investigated. In this study, the demonstration that DHA-OOH can induce apoptosis in the neuronal cells, for the first time, indicates DHA hydroperoxide as a potential neurotoxic product.

Several lines of evidence suggest that lipid peroxidation products represent potential intermediates for the oxidative modification of proteins under oxidative stress [31-35]. In our previous study [4], we characterized a specific monoclonal antibody 2B12 against SUL, which is an $N$-acyl-type (amide-linkage) adduct generated from the reaction of DHA-OOH with lysine residue, but not from the reaction of aldehydes with the amino residues in vitro. Recently, by using $\mathrm{mAb} 2 \mathrm{~B} 12$, we have observed an intensive SUL staining in the brain of Parkinson's patients (Maruyama, Osawa, unpublished data), as well as we found that SUL significantly accumulated in the mitochondrial fraction isolated from the DHA-OOH-treated cells (Data not shown). Therefore, we suggest not only loss of mitochondria membrane potential but also protein modification happened in the mitochondria in the DHA-OOH-treated cells.

Pathologically, other than apoptosis as a common causal or resulting feature, several disorders have been implicated in neurodegenerative diseases, such as $\beta$-amyloid aggregation and a progressive loss of dopaminergic neurons which are mostly characterized in AD and PD [36], respectively. Our further challenge is to investigate the influence of a DHA peroxidative product, namely DHA-OOH, on the other neurodegenerative disorders.

\section{Acknowledgement}

We thank Dr. Wakako Maruyama (National Institute for Longevity Science, Obu, Aichi, Japan) for her valuable advice.

\section{References}

[1] Porter, N.A., Caldwell, S.E., and Mills, K.A.: Mechanisms of free radical oxidation of unsaturated lipids. Lipids, 30, 277290, 1995.

[2] Gutteridge, J.M. and Halliwell, B.: The measurement and mechanism of lipid peroxidation in biological systems. Trends Biochem. Sci., 15, 129-135, 1990.

[3] Shibata, T., Iio, K., Kawai, Y., Shibata, N., Kawaguchi, M., Toi, S., Kobayashi, M., Kobayashi, M., Yamamoto, K., and Uchida, K.: Identification of a lipid peroxidation product as a potential trigger of the p53 pathway. J. Biol. Chem., 281, 1196-1204, 2006.

[4] Kawai, Y., Fujii, H., Okada, M., Tsuchie, Y., Uchida, K., and Osawa, T.: Formation of $N^{\varepsilon}$-(succinyl) lysine (SUL) in vivo: a novel marker for docosahexaenoic acid-derived protein modification. J. Lip. Res., 47, 1386-1398, 2006.

[5] Standstrom, P.A., Pardi, D., Tebbey, P.W., Dudek, R.W., Terrian, D.M., Folks, T.M., and Buttke, T.M.: Lipid hydroperoxide-induced apoptosis: lack of inhibition by Bcl-2 overexpression. FEBS Lett., 365, 66-70, 1995.

[6] Suraneni, V., Mahipal, K., Subhashini, J., Reddy, M.C., Reddy, M.M., Anilkumara, K., Roy, K.R., Reddy, G.V., and Reddanna, P.: Effect of 15-lipoxygenase metabolites, 15-(S)HPETE and15-(S)-HETE on chronic myelogenous leukemia cell line K-562: Reactive oxygen species (ROS) mediate caspase-dependent apoptosis. Biochem. Pharmacol., 74, 202-214, 2007.

[7] Bernoud-Hubac, N., Davies, S., Boutaud, O., Montine, T., and Roberts, II. L.: Formation of highly reactive gammaketoaldehydes (neuroketals) as products of the neuroprostane pathway. J. Biol. Chem., 276, 30964-30970, 2001.

[8] Forman, H.J. and Dickinson, D.A.: Introduction to serial reviews on 4-hydroxy-2-nonenal as a signaling molecule. Free Radic. Biol. Med., 37, 594-596, 2004.

[9] Lossi, L. and Gambino, G.: Apoptosis of the cerebellar neurons. Histo. Histophathol., 23, 367-380, 2008.

[10] Kato, Y., Mori, Y., Makino, Y., Morimitsu, Y., Hiroi, S., Ishikawa, T., and Osawa, T.: Formation of $N^{\varepsilon_{-}}$ (Hexanonyl)lysine in protein exposed to lipid hydroperoxide. J. Biol. Chem., 274, 20406-20414, 1999.

[11] Hui-Chun, Y., Tsai, A.-L., and Wang, L.-H.: Reaction mechanisms of 15-hydroperoxyeicosatetraenoic acid catalyzed by human prostacyclin and thromboxane synthases. Arc. Biochem. Biophys., 461, 159-168, 2007.

[12] Liu, X. and Osawa, T.: Cis astaxanthin and especially 9-cis astaxanthin exhibits a higher antioxidant activity in vitro compared to the all-trans isomer. Biochem. Biophys. Res. Commun., 357, 187-193, 2007.

[13] Pallotti, F. and Lenaz, G.: Isolation and subfractionation of mitochondria from animal cells and tissue culture lines. Methods Cell Biol., 80, 3-44, 2007.

[14] Ko, C.H., Shen, S.H., Yang, L.Y., Lin, C.W., and Chen, Y.C.: Gossypol reduction of tumor growth through ROS-dependent mitochondria pathway in human colorectal carcinoma cells. Int. J. Cancer, 121, 1670-1679, 2007.

[15] Andersen, J.K..: Does neuronal loss in Parkinson's disease 
involve programmed cell death?. BioEssays, 23, 640-646, 2001.

[16] Cutler, R.G., Kelly, J., Storie, K., Pedersen, W.A., Tammara, A., Hatanpaa, K., Troncoso, J.C., and Mattson, M.P.: Involvement of oxidative stress-induced abnormalities in ceramide and cholesterol metabolism in brain aging and Alzheimer's disease. Proc. Natl. Acad. Sci., 101, 2070-2075, 2004.

[17] Butterfield, D.A., Reed, T., Newman, SF., and Sultana, R.: Roles of amyloid beta-peptide-associated oxidative stress and brain protein modifications in the pathogenesis of Alzheimer's disease and mild cognitive impairment. Free Radic. Biol. Med., 43, 658-677, 2007.

[18] Osawa, T.: Nephroprotective and hepatoprotective effects of curcuminoids. Adv. Exp. Med. Biol., 595, 407-423, 2007.

[19] Yuan, H., Zheng, J.C., Liu, P., Zhang, S.F., Xu, J.Y., and Bai, L.M.: Pathogenesis of Parkinson's disease: oxidative stress, environmental impact factors and inflammatory processes. Neurosci. Bull., 23, 125-130, 2007.

[20] Montine, T.J. and Morrow, J.D.: Fatty acid oxidation in the pathogenesis of Alzheimer's disease. Am. J. Pathol., 166, 1283-1289, 2005.

[21] Tapiero, H., Ba, G.N., Couvreur, P., and Tew, K.D.: Polyunsaturated fatty acids (PUFA) and eicosanoids in human health and pathologies. Biomed. Pharmacother., 56, 215-222, 2002.

[22] Jian, W.Y., Arora, J.S., Oe, T., Shuvaev, V.V., and Blair, I.A..: Induction of endothelial cell apoptosis by lipid hydroperoxide-derived bifunctional electrophiles. Free Radic. Biol. Med., 39, 1162-1176, 2005.

[23] Maccarrone, M., Ranalli, M., Bellincampi, L., Salucci, M.L., Sabatini, S., Melino, G., and Alessandro, F.A.: Activation of different lipoxygenase isozymes induces apoptosis in human erythroleukemia and neuroblastoma cells. Biochem. Biophys. Res. Commun., 272, 345-350, 2000.

[24] Zhu, X., Su, B., Wang, X., Smith, M.A., and Perrya, G.: Causes of oxidative stress in Alzheimer disease. Cell. Mol. Life Sci., In press, 2007.

[25] Shirai, N. and Suzuki, H.: Effect of dietary docosahexaenoic acid and catechins on maze behavior in mice. Ann. Nutr. Metab., 48, 51-58, 2004.

[26] Kim, H.Y., Akbar, M., Lau, A., and Edsall, L.: Inhibition of neuronal apoptosis by docosahexaenoic acid (22:6n-3), Role of phosphatidylserine in antiapoptotic effect. J. Biol. Chem.,
275, 35215-35223, 2000.

[27] Rodriguez de., Turco, E.B., Belayev, L., Liu, Y., Busto, R., Parkins, N., Bazan, N.G., and Ginsberg, M.D.: Systemic fatty acid responses to transient focal cerebral ischemia: influence of neuroprotectant therapy with human albumin. J. Neurochem., 83, 515-524, 2002.

[28] Hashimoto, M., Shinozuka, K., Gamoh, S., Tanabe, Y., Hossain, M.S., Kwon, Y.M., Hata, N., Misawa, Y., Kunitomo, M., and Masumura, S.: The hypotensive effect of docosahexaenoic acid is associated with the enhanced release of ATP from the caudal artery of aged rats. J. Nutr., 129, 7076, 1999.

[29] Bazan, N.G., Palacios-Pelaez, R., and Lukiw, W.J.: Hypoxia signaling: significance in Alzheimer's disease. Mol. Neurobiol., 26, 283-298, 2002.

[30] Esterbauer, H., Schaur, R.J., and Zollner, H.: Chemistry and biochemistry of 4-hydroxynonenal, malondialdehyde and related aldehydes. Free Radic. Biol. Med., 11, 81-128, 1991.

[31] Shamo-Nagai, M., Maruyama, W., Kato, Y., Isobe, K., Tanaka, M., Naoi, M., and Osawa, T.: An inhibitor of mitochondrial complex I, rotenone, inactivates proteasome by oxidative modification and induces aggregation of oxidized proteins in SH-SY5Y cells. J. Neurosci. Res., 74, 589-597, 2003.

[32] Naoi, M., Maruyama, W., Shimoto-Nagai, M., Yi, H., Akao, Y., and Tanaka, M.: Oxidative stress in mitochondria: Decision of survival and death of neurons in neurodegenerative disorders. Molecular Neurobiology, 31, 81-93, 2005.

[33] Montine, K.S., Quinn, J.F., Zhang, J., Fessel, J.P., Roberts II, L.J., Morrow, J.D., and Montine, T.J.: Isoprostanes and related products of lipid peroxidation in neurodegenerative disease. Chem. Phys. Lip., 128, 117-124, 2004.

[34] Negre-Salvayre, A., Coatrieus, C., Ingueneau, C., and Salvayre, R.: Advanced lipid peroxidation end products in oxidative damage to proteins. Potential role in diseases and therapeutic prospects for the inhibitors. Bri. J. Pharmacol., In press, 2007.

[35] Haddad, J.J.: Redox and oxidant-mediated regulation of apoptosis signaling pathways: immuno-pharmaco-redox conception of oxidative siege versus cell death commitiment. Int. Immunopharmacol., 4, 475-493, 2004.

[36] Lin, M.T. and Flint Beal, M.: Mitochondrial dysfunction and oxidative stress in neurodegenerative diseases. Nature, $\mathbf{4 3 3}$, 787-795, 2006. 